\title{
Número de progenie y exactitud de la valoración genética en características productivas de alpacas. Un estudio de simulación
}

\author{
Number of progeny number and accuracy of breeding value in productive \\ traits of alpacas. A simulation study
}

\author{
Gerardo C. Mamani M. ${ }^{1,2}$, Mario L. Gonzales C. ${ }^{1}$
}

\section{Resumen}

\begin{abstract}
El objetivo del estudio fue evaluar mediante simulación las exactitudes de la predicción del valor de cría según la heredabilidad de la característica y el número de progenie. Se simularon poblaciones con seis números de progenie para machos $(n=15,30,50,75$, 100 y 150$)$ y tres para hembras $(\mathrm{n}=1,2$ y 3$)$ y características con tres heredabilidades $\left(\mathrm{h}^{2}\right.$ $=0.098,0.22$ y 0.56). La predicción de los valores de cría fue mediante el método del mejor predictor lineal insesgado y la exactitud fue calculada a partir de la diagonal de la matriz de la ecuación de los modelos mixtos. Se obtuvo que a mayor heredabilidad la exactitud fue también mayor en todos los escenarios. Respecto al número de hijos por reproductor, se obtuvo valores encima de 0.9 de exactitud cuando la progenie fue mayor a 30 hijos. En el caso de las hembras, las mayores exactitudes fueron para la heredabilidad de 0.56 con valores de $0.71,0.74$ y 0.76 para 1,2 y 3 hijos, respectivamente. La exactitud de la predicción del valor de cría en características con heredabilidad similar a las de importancia en alpacas fue mayor cuando se incrementó el número de hijos por padre y cuando la heredabilidad de la característica fue mayor.
\end{abstract}

Palabras clave: valor de cría, exactitud, heredabilidad, alpaca

\footnotetext{
${ }^{1}$ Estación Experimental Agraria Illpa, Dirección de Recursos Genéticos y Biotecnología, Instituto Nacional de Innovación Agraria (INIA), Puno, Perú

${ }^{2}$ E-mail: ct_illpa1@inia.gob.pe
}

Recibido: 9 de noviembre de 2020

Aceptado para publicación: 20 de mayo de 2021

Publicado: 24 de agosto de 2021

CLos autores. Este artículo es publicado por la Rev Inv Vet Perú de la Facultad de Medicina Veterinaria, Universidad Nacional Mayor de San Marcos. Este es un artículo de acceso abierto, distribuido bajo los términos de la licencia Creative Commons Atribución 4.0 Internacional (CC BY 4.0) [https:// creativecommons.org/licenses/by/4.0/deed.es] que permite el uso, distribución y reproducción en cualquier medio, siempre que la obra original sea debidamente citada de su fuente original 
The aim of this study was to evaluate by simulation the accuracy of the prediction of the breeding value according to the heritability of the trait and the number of progenies. Populations were simulated with six progenies for males $(n=15,30,50,75,100$ and $150)$ and three for females $(n=1,2$ and 3$)$ and characteristics with three heritability $\left(h^{2}=\right.$ $0.098,0.22$ and 0.56 ). The prediction of the breeding values was calculated by the method of the best unbiased linear predictor and the accuracy was calculated from the diagonal of the matrix of the mixed model equation. It was found that the higher heritability the accuracy was also higher in all scenarios. Regarding the number of progenies per male, accuracy values above 0.9 were obtained when the progeny was greater than 30 . In the case of females, the highest accuracies were 0.56 for heritability with values of $0.71,0.74$ and 0.76 for 1, 2 and 3 offspring, respectively. The accuracy of the prediction of the breeding value in traits with heritability similar to those of importance in alpacas was greater when the number of progenies per male increased and when the heritability of the trait was greater.

Key words: estimated breeding value, accuracy, heritability, alpaca

\section{INTRODUCCIÓN}

En mejoramiento genético, la selección de individuos genéticamente superiores es importante para designarles como reproductores del rebaño. La predicción de los valores de cría (EBV) mediante el método del mejor predictor lineal insesgado (BLUP, best linear unbiased prediction) se aplica ampliamente en diferentes especies domésticas (Gianola y Rosa, 2015). Los EBV proveen un estimado del potencial genético de un animal y es expresado en relación con el promedio de la población. Estas predicciones van acompañadas de la exactitud, las cuales ayudan a identificar el error y la incerteza de la predicción (Tier et al., 1990)

La exactitud indica el grado de confiabilidad del valor cría. A mayor exactitud menor cambio posible del valor de cría. Esta exactitud mejora cuando el animal evaluado tiene mas información fenotípica y de genealogía (Clark et al., 2012). Sin embargo, un criador de alpacas se puede preguntar ¿Cuántos descendientes debe tener un reproductor para evaluarlo adecuadamente?, respuesta que por el momento se desconoce dicho número mínimo de progenie que permita tener una exactitud confiable de la predicción del valor de cría.

La alpaca es una especie de importancia para la ganadería altoandina del Perú, pero existen muy pocos programas de mejora genética, generalmente de entidades privadas (Gutiérrez et al., 2009), de allí que sea necesario estudiar los temas acerca de la valoración genética en esta especie para las evaluaciones genéticas. El objetivo de este estudio fue evaluar, mediante una simulación, las exactitudes de la predicción del valor de cría según la heredabilidad de la característica y el número de progenie.

\section{Materiales y Métodos}

\section{Población Simulada}

Los datos fenotípicos y de pedigrí fueron simulados usando el software QMSim (Sargolzaei y Schenkel, 2014). En el proceso 
de simulación, la población histórica consistió en 100 generaciones con una gradual disminución del tamaño de 3000 a 640 individuos. Un total de 600 hembras y 40 machos de la ultima generación de la población histórica fueron los fundadores de la población reciente. La población fue expandida por 10 generaciones basadas en selección fenotípica, donde la progenie tenía $50 \%$ de probabilidad de ser macho, siendo de $60 \%$ el reemplazo de machos y de $20 \%$ para hembras. Se simularon diferentes escenarios para lograr que el número de progenie sea de 1, 2, 3 por cada hembra, y 15, $30,50,75,100,150$ por cada macho.

Las heredabilidades usadas para la simulación se basaron en estimaciones reales del diámetro de fibra (0.561, Cruz et al., 2020), peso de vellón (0.098, Gutiérrez et al., 2009) y porcentaje de medulación $(0.225$, Cruz et al., 2019) en alpacas.

\section{Análisis Estadístico}

Las predicciones de los valores de cría se realizaron mediante el mejor predictor lineal insesgado (BLUP, Best linear unbiased prediction) (Henderson, 1976). Esta metodología integra datos de pedigrí y de desempeño. El modelo lineal mixto en la notación matricial fue el siguiente: $y=X b+Z a+e$, donde $y$ es el vector de observaciones, $X$ es la matriz de incidencia de los efectos fijos, $Z$ es la matriz de incidencia que relaciona las observaciones a los efectos genéticos aditivos, $b$ es el vector de los efectos fijos, $a$ es el vector de los efectos genéticos aditivos y $e$ es vector de los residuales. Se asumió que los efectos aleatorios eran independientes y normalmente distribuidos: $a \sim N\left(0, A \sigma^{2}\right), y e$ $\sim N\left(0, I \sigma^{2}\right)$, donde $A$ es la matriz del numerador de parentesco, $I$ es la matriz identidad, $\sigma^{2}{ }_{a}$ es la varianza genética aditiva, y $\sigma_{e}^{2}$ es la varianza residual.

La exactitud de Mrode (2014) fue usada para calcular la exactitud $\left(a c c_{i}\right)$ según:

$$
a c c_{i}=\sqrt{1-\frac{P E V_{i}}{\sigma_{a}^{2}}}
$$

donde $P E V_{i}$ es la varianza del error de predicción, $\sigma^{2}{ }_{a}$ es la varianza genética aditiva de la característica analizada.

Los valores de cría y las exactitudes fueron calculados usando los programas de la familia BLUPF90 (Misztal et al., 2002).

\section{Resultados}

Se calcularon los valores de cría y sus respectivas exactitudes de reproductores con seis tamaños de progenie $(15,30,50,75,100$ y 150) y para tres características con diferentes heredabilidades $\left(\mathrm{h}^{2}=0.56,0.225 \mathrm{y}\right.$ 0.098). Los valores de exactitud variaron según la heredabilidad de la característica y el número de progenie de cada reproductor (Cuadro 1). En los reproductores con información propia, las mayores exactitudes se observaron con la heredabilidad de 0.56 y las menores, cuando la heredabilidad fue de 0.098. Así mismo, las exactitudes se incrementaron de 0.87 a $0.98,0.78$ a 0.96 y 0.65 a 0.89 cuando el número de progenie varió de 15 a 150 hijos para las características con heredabilidades de $0.56,0.225 \mathrm{y}$ 0.098 , respectivamente.

La exactitud aumenta rápidamente con el aumento del número de la progenie hasta llegar a un zenit (Figura 1). Si el interés es evaluar reproductores para características con alta heredabilidad $\left(\mathrm{h}^{2}=0.56\right.$; por ejemplo, diámetro medio de fibra), sería suficiente medir a 15 hijos para tener una exactitud mayor a 0.8 ; en cambio al evaluar una característica con heredabilidad baja $\left(\mathrm{h}^{2}=0.098\right.$, por ejemplo, peso de vellón sucio) se requerirá 30 hijos para alcanzar una exactitud mayor a 0.8 .

Las exactitudes son menores cuando el reproductor no tiene información propia (Cuadro 2) frente a los reproductores que la tienen (Cuadro 1). Sin embargo, estas diferencias se reducen conforme aumenta el número de progenie, llegando a ser casi iguales 
Cuadro 1. Promedio y desviación estándar (DE) de la exactitud de la predicción de valores genéticos en alpacas de acuerdo con la heredabilidad de la característica y el número de progenie cuando el reproductor tiene datos propios

\begin{tabular}{|c|c|c|c|c|c|c|c|}
\hline \multirow{2}{*}{$\begin{array}{l}\text { Reproductores } \\
\text { (n) }\end{array}$} & \multirow{2}{*}{$\begin{array}{l}\text { Progenie por } \\
\text { reproductor }\end{array}$} & \multicolumn{2}{|c|}{$\mathrm{h}^{2}=0.56$} & \multicolumn{2}{|c|}{$\mathrm{h}^{2}=0.22$} & \multicolumn{2}{|c|}{$\mathrm{h}^{2}=0.098$} \\
\hline & & Promedio & $\mathrm{DE}$ & Promedio & $\mathrm{DE}$ & Promedio & $\mathrm{DE}$ \\
\hline 40 & 15 & 0.87 & 0.010 & 0.78 & 0.018 & 0.65 & 0.026 \\
\hline 40 & 30 & 0.92 & 0.004 & 0.86 & 0.010 & 0.74 & 0.018 \\
\hline 12 & 50 & 0.94 & 0.003 & 0.89 & 0.005 & 0.79 & 0.016 \\
\hline 8 & 75 & 0.96 & 0.002 & 0.92 & 0.005 & 0.83 & 0.013 \\
\hline 12 & 100 & 0.97 & 0.001 & 0.94 & 0.004 & 0.86 & 0.010 \\
\hline 8 & 150 & 0.98 & 0.001 & 0.96 & 0.003 & 0.89 & 0.010 \\
\hline
\end{tabular}

Cuadro 2. Promedio y desviación estándar (DE) de la exactitud de la predicción de valores genéticos en alpacas de acuerdo con la heredabilidad de la característica y el número de progenie cuando el reproductor no tiene datos propios

\begin{tabular}{ccccccccc}
\hline \multirow{2}{*}{$\begin{array}{c}\text { Reproductores } \\
\text { (n) }\end{array}$} & Progenie por & \multicolumn{2}{c}{$\mathrm{h}^{2}=0.56$} & \multicolumn{2}{c}{$\mathrm{h}^{2}=0.22$} & \multicolumn{2}{c}{$\mathrm{h}^{2}=0.098$} \\
\cline { 3 - 8 } & reproductor & Promedio & DE & Promedio & DE & Promedio & DE \\
\hline 40 & 15 & 0.84 & 0.015 & 0.75 & 0.023 & 0.61 & 0.029 \\
40 & 30 & 0.90 & 0.006 & 0.84 & 0.011 & 0.71 & 0.019 \\
12 & 50 & 0.93 & 0.004 & 0.88 & 0.006 & 0.77 & 0.018 \\
8 & 75 & 0.95 & 0.002 & 0.92 & 0.006 & 0.82 & 0.015 \\
12 & 100 & 0.97 & 0.001 & 0.94 & 0.004 & 0.86 & 0.010 \\
8 & 150 & 0.98 & 0.001 & 0.96 & 0.003 & 0.89 & 0.009 \\
\hline
\end{tabular}

Cuadro 3. Promedio y desviación estándar (DE) de la exactitud de la predicción de valores genéticos en alpacas de acuerdo con la heredabilidad de la característica y el número de progenie cuando la hembra tiene datos propios

\begin{tabular}{cccccccc}
\hline \multirow{2}{*}{$\begin{array}{c}\text { Madres } \\
(\mathrm{n})\end{array}$} & $\begin{array}{c}\text { Progenie por } \\
\text { hembra }\end{array}$ & \multicolumn{2}{c}{$\mathrm{h}^{2}=0.56$} & \multicolumn{2}{c}{$\mathrm{h}^{2}=0.22$} & \multicolumn{2}{c}{$\mathrm{h}^{2}=0.098$} \\
\cline { 3 - 8 } & 1 & Promedio & $\mathrm{DE}$ & Promedio & $\mathrm{DE}$ & Promedio & $\mathrm{DE}$ \\
\hline 600 & 2 & 0.71 & 0.044 & 0.58 & 0.068 & 0.43 & 0.081 \\
600 & 3 & 0.76 & 0.029 & 0.64 & 0.047 & 0.50 & 0.059 \\
600 & 0.78 & 0.021 & 0.67 & 0.036 & 0.53 & 0.048 \\
\hline
\end{tabular}




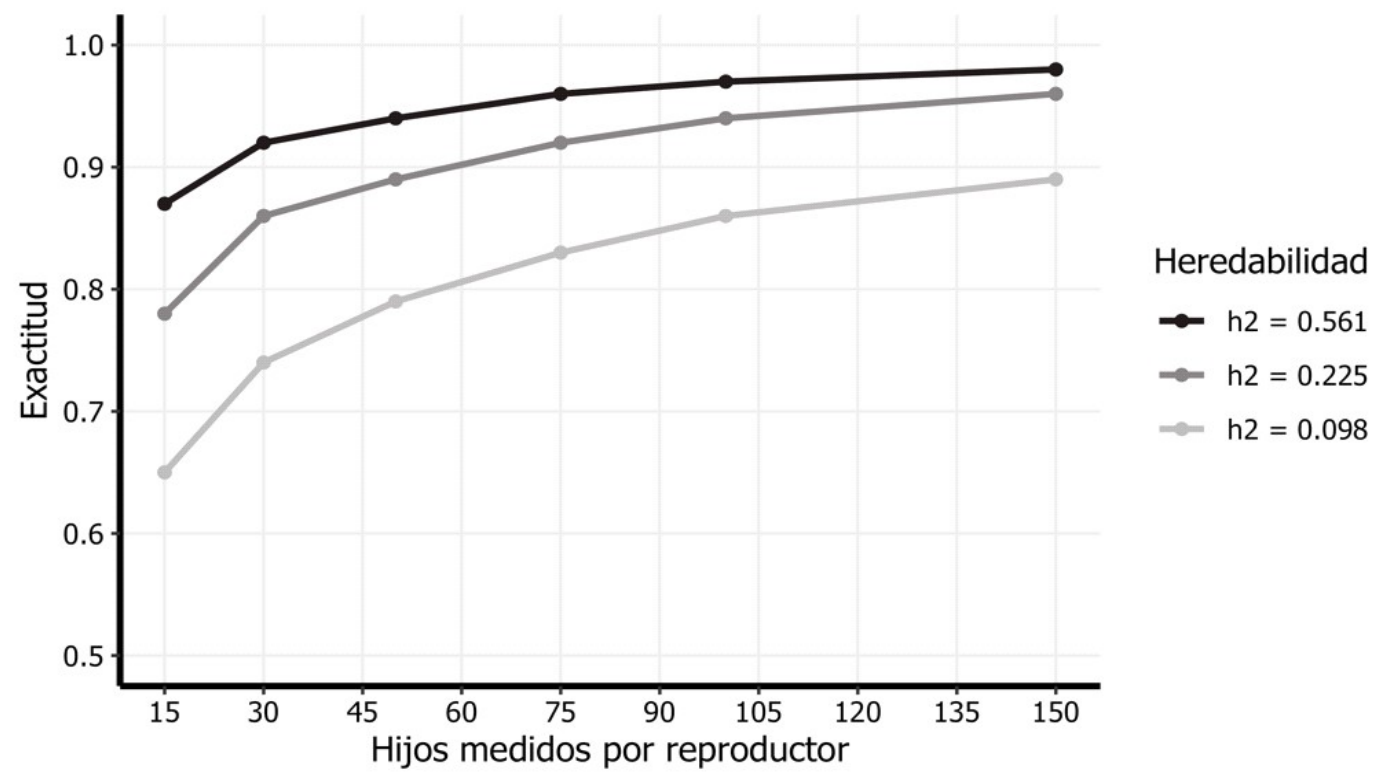

Figura 1. Exactitud de la predicción del valor de cría de reproductores alpacas según el número de progenie y la heredabilidad de la característica considerada

cuando la progenie alcanza a 100 descendientes por reproductor. En el caso de las hembras (Cuadro 3), que naturalmente pueden tener menos progenie, el comportamiento es similar al caso de los machos. A mayor heredabilidad de la característica y mayor número de progenie, la exactitud también se incrementa.

\section{Discusión}

La exactitud de la predicción del valor de cría es un parámetro crítico al realizar la selección de reproductores porque influye directamente en el progreso genético (Falconer y Mackar, 1996). En el presente estudio se observó que la exactitud de las predicciones aumenta con el incremento del número de hijos del reproductor evaluado. Estos resultados concuerdan con lo reportado por Mueller (2018), quien para una ca- racterística con heredabilidad de $0.5,22$ hijos por padres permitieron alcanzar una exactitud de 0.8. Asimismo, Mueller y Paz (1992), en una prueba de progenie de carneros Merino encontraron exactitudes de 0.86 a 0.96 cuando el número de hijos varió de 36 a 106, siendo la heredabilidad de 0.40 . A mayor información esté disponible relacionada con la genética de un animal, más exacta será la exactitud de la predicción del valor de cría.

La información sobre la descendencia es valiosa porque la descendencia recibe la mitad de los genes del animal. Si se dispone de un número suficiente de crías, se puede cuantificar el efecto de muestreo mendeliano (Hill y Weir, 2011) y se puede estimar con mayor precisión el valor de cría del animal (Oldenbroek y van der Waaij, 2014). La diferencia de exactitud puede ser el resultado de una heredabilidad diferente (Falconer y Mackay, 1996), lo cual fue corroborado en el presente estudio donde para una heredabilidad 
de 0.56 , las exactitudes promedio fueron superiores en todos los escenarios a las exactitudes de heredabilidades de 0.22 y estas a las de 0.098 .

\section{Conclusiones}

La exactitud de la predicción del valor de cría en características con heredabilidad similar a las de importancia en alpacas fue mayor cuando se incrementó el número de hijos por padre y cuando la heredabilidad de la característica fue mayor.

\section{Agradecimientos}

Al Grupo de Mejoramiento Genético de la Facultad de Zootecnia e Ingeniería de Alimentos de la Universidad de Sao Paulo por el servidor para las simulaciones y el análisis.

\section{Literatura Citada}

1. Clark SA, Hickey JM, Daetwyler HD, van der Werf JH. 2012. The importance of information on relatives for the prediction of genomic breeding values and the implications for the makeup of reference data sets in livestock breeding schemes. Genet Sel Evol 44: 1-9. doi: 10.1186/1297-9686-44-4

2. Cruz A, Menéndez-Buxadera A, Gutiérrez, G, Morante R, Burgos A, Gutiérrez JP. 2020. Genetic (co)variance across age of fiber diameter and standard deviation in Huacaya alpacas, estimated by repeatability, multi-trait and random regression models. Livest Sci 231: 103863. doi: 10.1016/j.livsci.2019.103863

3. Cruz A, Morante R, Gutiérrez JP, Torres R, Burgos A, Cervantes I. 2019. Genetic parameters for medullated fiber and its relationship with other productive traits in alpacas. Animal 13: 1358-1364. doi: $10.1017 /$ S1751731118003282
4. Falconer DS, Mackay TFC. 1996. Introduction to quantitative genetics. $4^{\text {th }}$ ed. Essex, UK: Longman Group. 448 p.

5. Gianola D, Rosa GJ. 2015. One hundred years of statistical developments in animal breeding. Annu Rev Anim Biosci 3: 19-56. doi: 10.1146/annurevanimal-022114-110733

6. Gutiérrez JP, Goyache F, Burgos A, Cervantes I. 2009. Genetic analysis of six production traits in Peruvian alpacas. Livest Sci 123: 193-197. doi: 10.1016/ j.livsci.2008.11.006

7. Henderson CR. 1976. A rapid method for computing the inverse of a relationship matrix. J. Dairy Sci 58:1727-1730. doi: 10.3168/jds.S0022-0302(75)84776-X

8. Hill WG, Weir BS. 2011. Variation in actual relationship as a consequence of Mendelian sampling and linkage. Genet Res 93 :47-64. doi: 10.1017/S0016672310000480

9. Misztal I, Tsuruta S, Strabel T, Auvray B, Druet T, Lee DH. 2002. BLUPF90 and related programs (BGF90). In: VII World Congress on Genetics Applied to Livestock Production. Montpellier, France.

10. Mrode RA. 2014. Linear models for the prediction of animal breeding values, $3^{\text {rd }}$ ed. Oxfordshire, UK: CABI.

11. Mueller JP. 2018. Aspectos teóricos en el diseño de pruebas de progenie. Comunicación Técnica N. ${ }^{\circ} 744$. Ediciones INTA. Argentina. [Internet]. Disponible en https://repositorio.inta.gob.ar/handle/ 20.500.12123/3788\#

12. Mueller JP, Paz AP. 1992. Prueba de progenie para carneros Merino Australiano en un establecimiento de la Patagonia Argentina. En: Congreso Mundial de Ovinos y Lanas. Buenos Aires, Argentina.

13. Oldenbroek K, van der Waaij L. 2014. Textbook animal breeding: animal breeding and genetics for BSc students. The Netherlands: Wageningen University. $311 \mathrm{p}$. 
14. Sargolzaei M, Schenkel FS. 2009. QMSim: a large-scale genome simulator for livestock. Bioinformatics 25: 680-681. doi: 10.1093/bioinformatics/btp045
15. Tier B, Schneeberger M, Hammond K. 1990. Accuracy of estimated breeding value. In: Proc. $8^{\text {th }}$ Conf. AAABG, Hamilton and Palmerston North. New Zealand. 